\title{
Processing Nested Complex Sequence Pattern Queries over Event Streams
}

\author{
Mo Liu, Medhabi Ray, Elke A. Rundensteiner, Daniel J. Dougherty \\ Worcester Polytechnic Institute, Worcester, MA 01609, USA \\ (liumo|medhabi|rundenst $\mid$ dd)@cs.wpi.edu \\ Chetan Gupta, Song Wang, Ismail Ari‡, Abhay Mehta \\ USA Hewlett-Packard Labs, USA \\ ${ }^{\ddagger}$ Ozyegin University, Turkey \\ (chetan.gupta|songw|abhay.mehta)@hp.com ¥Ismail.Ari@ozyegin.edu.tr
}

\begin{abstract}
Complex event processing (CEP) has become increasingly important for tracking and monitoring applications ranging from health care, supply chain management to surveillance. These monitoring applications submit complex event queries to track sequences of events that match a given pattern. As these systems mature the need for increasingly complex nested sequence queries arises, while the state-of-the-art CEP systems mostly focus on the execution of flat sequence queries only. In this paper, we now introduce an iterative execution strategy for nested CEP queries composed of sequence, negation, AND and OR operators. Lastly we have introduced the promising direction of applying selective caching of intermediate results to optimize the execution. Our experimental study using real-world stock trades evaluates the performance of our proposed iterative execution strategy for different query types.
\end{abstract}

\section{Categories and Subject Descriptors}

H.4.2 [Information Systems]: Database Management Systems [Query Processing]

\section{INTRODUCTION}

Complex event processing (CEP) has become increasingly important in modern applications, ranging from supply chain management for RFID tracking to real-time intrusion detection [1,2, 3]. CEP must be able to support sophisticated pattern matching on real time event streams including the arbitrary nesting of sequence operators and the flexible use of negation in such nested sequences. For example, consider reporting contaminated medical equipments in a hospital $[4,5]$. Let us assume that the tools for medical operations are RFID-tagged. The system monitors the histories of the equipment (such as, records of surgical usage, of washing, sharpening and disinfection). When a healthcare worker puts a box of surgical tools into a surgical table equipped with RFID readers, the computer would display approximate warnings such as "This tool must be disposed". A query $Q_{1}=S E Q$ (Recycle $r$, Washing $w$, NOT SEQ(Sharpening s, Disinfection d, Checking c), Operating $o p$ ) with the condition that ([ID] (equality on ID) and op.ins-type = "surgery") expresses this critical condition that after being re-

Permission to make digital or hard copies of all or part of this work for personal or classroom use is granted without fee provided that copies are not made or distributed for profit or commercial advantage and that copies bear this notice and the full citation on the first page. To copy otherwise, to republish, to post on servers or to redistribute to lists, requires prior specific permission and/or a fee.

DMSN '10, September 13, 2010, Singapore

Copyright 2010 ACM 978-1-4503-0416-0 ...\$5.00. cycled and washed, a surgery tool is being put back into use without first being sharpened, disinfected and then checked for quality assurance. Such complex sequence queries contain complex negation specifying the non-occurrence of composite event instances, such as negating the composite event of sharpened, disinfected and checked subsequences.

However, the state-of-the-art CEP in the literature including SASE [1] and ZStream [3] do not support such nested queries. Even though the Cayuga system [2] mentions composable queries, they assume the negation filter is only applied to a single primitive event type within the SEQ pattern. Our objective however is to allow the specification of negation within any level of the nested query as in the above example. While CEDR [6] allows applying negation over composite event types within their proposed language, the execution strategy for such nested queries is not discussed. In short, no processing mechanisms for nested complex negation of CEP queries have been discussed in the literature to date. In this work, we address this gap by designing an execution strategy specifically to handle nested CEP queries specified by the nested complex expression query language NEEL ${ }^{1}$. The semantics of this language is presented in [7].

Our contributions in this paper include:

- We introduce an algebraic query plan for nested CEP queries expressed in NEEL [7].

- We design an iterative topdown execution strategy for NEEL queries that applies a window constraint tightening technique designed to correctly process nested sub-queries. Intermediate results are pushed up conservatively for delayed resolution when a child query can't be fully answered locally for nested negation.

- We experimentally evaluate our proposed execution strategy on real data streams studying the impact of different properties of nested queries, including sub-query lengths and nesting levels.

- Lastly selective caching of intermediate results is introduced as a strategy for optimizing the execution. Caching is shown to be extremely effective at improving the query processing performance.

\section{NESTED CEP QUERY MODEL}

\subsection{Event Model}

An event instance is an occurrence of interest which can be either primitive or composite as further introduced below. A primitive

${ }^{1}$ NEEL stands for Nested Complex Event Query Language. 
event instance denoted by a lower-case letter (e.g., 'e') is the smallest, atomic occurrence of interest in a system. $e_{i}$.ts and $e_{i}$.te denote the start and the end timestamp of an event instance $e_{i}$, respectively, with $e_{i}$.ts $\leq e_{i}$.te. For a primitive event instance e, $e_{i}$.ts $=e_{i}$.te. For simplicity, we use the subscript $\mathrm{i}$ attached to a primitive instance $e$ to denote the timestamp $i$.

A composite event instance is composed of constituent primitive event instances $e=\left\langle e_{1}, e_{2}, \ldots, e_{n}\right\rangle$. A composite event instance e occurs over an interval. The start and end timestamps of e are equal to $\min \left\{e_{i}\right.$.ts $\mid e_{i}$ in e $\}$ and $\max \left\{e_{i}\right.$.te $\mid e_{i}$ in e $\}$, respectively.

An event type is denoted by a capital letter, say $E_{i}$. An event type $E_{i}$ describes a set of attributes that the event instances of this type share. An event type can be either a primitive or a composite event type [8]. Primitive event types are pre-defined in the application domain of interest. Composite event types are aggregated event types created by combining other primitive and/or composite event types. $e_{i} \in E_{j}$ denotes that $e_{i}$ is an instance of the type $E_{j}$. Suppose one of the attributes of $E_{j}$ is attr and $e_{i} \in E_{j}$, then we use $e_{i}$.attr to denote $e_{i}$ 's value for that attribute.

\subsection{The Nested Complex Pattern Query Language NEEL}

We now briefly introduce the NEEL query language for specifying complex nested event pattern queries $[1,6,9]$ as an extension of basic non-nested languages from the literature. NEEL supports the nesting of AND, OR, Negation and SEQ operators at any query nesting level as in Table 1.

\begin{tabular}{|c|c|}
\hline & $\begin{aligned} &<\text { Query }>::= \text { PATTERN }<\text { event-expression }> \\
& \text { WITHIN }<\text { window }> \\
& {[\text { RETURN }<\text { set of primitive events }>] } \\
&<\text { event-expression }>=<\text { ex }>\end{aligned}$ \\
\hline & $\begin{array}{l}<\operatorname{ex}>::= \\
\operatorname{SEQ}\left((<\mathrm{ex}>\mid !(<\mathrm{ex}>,[<\mathrm{q}>]))^{*},<\mathrm{ex}>,(<\mathrm{ex}>\mid\right. \\
\left.!(<\mathrm{ex}>,[<\mathrm{q}>]))^{*},[<\mathrm{q}>]\right) \\
\mid \mathbf{A N D}\left(\left(<\mathrm{ex}>,(<\mathrm{ex}>\mid !(<\mathrm{ex}>,[<\mathrm{q}>]))^{*},[<\mathrm{q}>]\right)\right. \\
\mathbf{O R}\left((<\mathrm{ex}>)^{+},[<\mathrm{q}>]\right) \\
(<\text { primitive-event type }>,[<\text { var }>])\end{array}$ \\
\hline & $\begin{array}{l}<\text { primitive-event type }>::=E_{1}\left|E_{2}\right| \ldots \\
<\text { var }>::=\text { event variable } e_{i} \\
<\mathrm{q}>::=(<\text { elemqual }>)^{*} \\
<\text { elemqual }>::=<\text { var }>\text {.attr }<\text { op }><\text { var }>\text {.attr } \mid \\
\quad<\text { var }>\text {.attr }<\text { op }>\text { constant } \\
<\text { op }>::=<|>| \leq|\geq|=\mid != \\
<\text { window }>::=\text { time duration } \mathrm{w} \mid \text { tuple count } \mathrm{c}\end{array}$ \\
\hline
\end{tabular}

Table 1: NEEL Query Language

A primitive event type $E_{i}$ itself is an event expression. If $E_{1}, E_{2}$ ,..., $E_{n}$ are event expressions, an application of SEQ, AND and OR over these event expressions is again an event expression [8]. In other words, nesting of AND, OR and SEQ operators is supported.

SEQ in the PATTERN clause specifies a particular order in which the event instances of interest should occur. If there is a ! (NOT) symbol before an event expression in an operator, we say that the event expression marked by ! is to be negated. Event instances that satisfy the positive components with no events in the stream relative to this match satisfying the negative components are output. If several adjacent event types are marked by ! in a SEQ operator such as $\operatorname{SEQ}\left(E_{1}, ! E_{2}, ! E_{3}, E_{4}\right)$, the query requires the nonexistence of any $E_{2}$ and $E_{3}$ events in either order between $E_{1}$ and $E_{4}$ events within the input stream. In other words $\left\langle e_{1}, e_{3}, e_{4}\right\rangle$ and $\left\langle e_{1}, e_{2}, e_{4}>,<e_{1}, e_{3}, e_{2}, e_{4}>\right.$ and $<e_{1}, e_{2}, e_{3}, e_{4}>$ all match for this query.

An event expression $\exp _{i}$ can be used as a component in SEQ, $\mathrm{AND}$ and OR operators to construct another expression $\exp _{j}$. Then we call $\exp _{j}$ the outer or parent expression of $\exp _{i}$ and $\exp _{i}$ the inner (or child) expression of $\exp _{j}$. Qualification in the PATTERN clause contains predicates on single attributes or on attributes across multiple event types in the query $[6,1]$. The event variables defined in an outer expression are visible within the scope of its own nested inner expressions. Local predicates are specified directly inside $\exp _{i}$. Correlated predicates involving events from both an outer and an inner expression are associated with the innermost expression that defines an event in the predicate. Correlated predicates involving two adjacent sibling expressions are not allowed since the events in one inner expression are not visible in any sibling.

The WITHIN clause indicates the temporal interval within which the event instances of interest must occur. The RETURN clause transforms the set of matching event instances extracted by the query into a complex event as specified in the output specification.

$Q_{1}$ below in Figure 1 is a sample query expressed by $N E E L$.

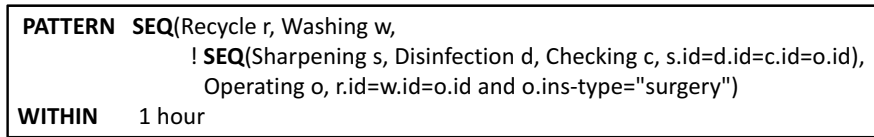

Figure 1: Sample Query $Q_{1}$ for Hospital Hygiene

\subsection{Nested CEP Query Plan}

A query expressed by a NEEL specification is translated into a default algebraic query plan composed of the following algebraic operators: Window Sequence (WinSeq), Window Or (WinOr) and Window And (WinAnd). During query transformation, each expression in the event pattern is mapped to one operator node in the query plan. The same window $w$ is assigned to all operator nodes. WinSeq first extracts all matches to the positive components specified in the query, and then filters out events based on negative components as specified in the query. WinOr returns an event $e$ if $e$ matches any one of the event expressions specified in the WinOr operator. WinAnd computes the cross product of its positive components. For queries expressed by NEEL, predicates are placed into the respective algebra operators in the nested event expressions (see Section 2.2).

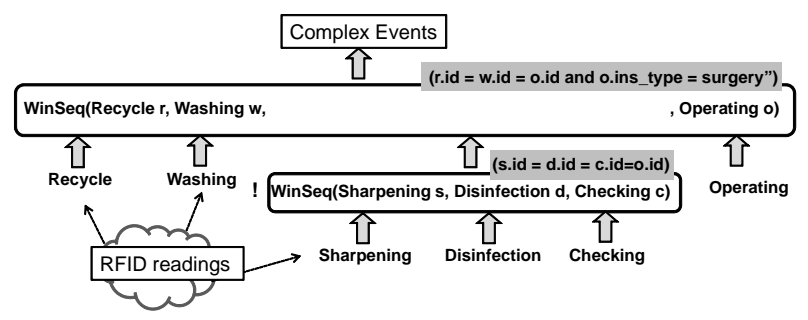

Figure 2: Basic Query Plan

EXAMPLE 1. Figure 2 depicts the query plan for query $Q_{1}$ in Figure 1. The two SEQ expressions in $Q_{1}$ are transformed into two WinSeq operator nodes in the plan. The predicate $s . i d=d . i d=c . i d$ $=o . i d$ is placed with the inner WinSeq operator node containing the negative component. The other predicates are attached to the topmost WinSeq operator node.

\section{NESTED CEP QUERY PROCESSING}

\subsection{Execution of Individual Operators}

For simplicity, we briefly review the implementation strategy of one of the operators, namely, the SEQ operator, while the others can be implemented in a similar fashion. We adopt the state-of-the-art stack-based strategy for SEQ execution $[1,10,11]$. We associate a stack with each event type in the query. Each received event instance is simply appended to the end of the stack of its type. Event instances are augmented with pointers $p t r_{i}$ to adjacent events to facilitate quick locating of related events in other stacks during result construction. 
The arrival of an event instance $e_{m}$ of the last event type $E_{m}$ of a query $q_{i}$ triggers the compute function of $q_{i}{ }^{2}$. The result construction is done by a depth first search along instance pointers $p t r_{i}$ rooted at that last arrived instance $e_{m}$. All paths composed of edges "reachable" by that root $e_{m}$ correspond to one matching event sequence returned for $q_{i}$. When negative event types are specified in WinSeq, then during sequence construction any edges "reachable" from the root $e_{m}$ are skipped if an instance of the negative event type is found in the corresponding stream position. Events that are outdated based on the window constraints are purged.

\subsection{Iterative Nested Execution Strategy}

Following the principle of nested query execution for SQL queries $[12,13,14,15]$, the outer query is evaluated first followed by its inner sub-queries. The results of the inner queries are passed up and joined with the results of the outer query. The main idea of our nested execution is about passing down more stringent window constraints from outer queries to inner queries. For every outer partial query result, a constraint window (see Figure 3 ) is passed down for processing each of its children sub-queries. These sub-queries compute results involving events within the substream constrained by the constraint window. Qualified result sequences of the inner operators are passed up to the parent operator and the outer operator then joins its own local results with that of its positive sub-queries. The outer sequence result is filtered if the result set of any of its negative sub-queries is not empty. We apply iterative execution until a final result sequence is produced by the root operator. Finally, the process repeats when the outer query consumes the next instance $e$. We will discuss nested queries with negation and predicates in more detail in Sections 3.3 and 3.4, respectively.

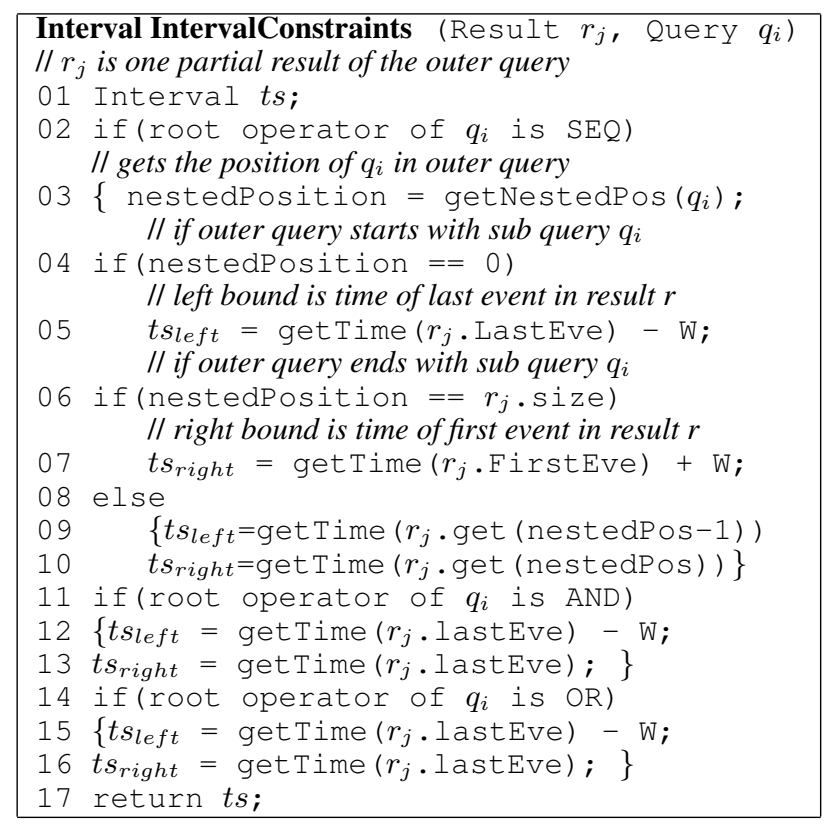

Figure 3: Algorithm to Compute Interval Constraints for an Inner Query $Q_{i}$ Given an Outer Partial Result $r_{j}$

\subsection{Processing Nested Queries with Negation}

We now describe our approach of supporting negations in nested queries. In SASE $[1,11,10]$, flat queries can have negations and they are dealt with using the timestamp information. More precisely, if a query has a negative A between positive B and C event types, they first evaluate the query without the negation, i.e., they

${ }^{2}$ if $E_{m}$ is a negative event type, postponed sequence evaluation is applied. We omit the details here. compute all B-C pairs. Then for every result generated they check if an $\mathrm{A}$ event occurred between the qualified $\mathrm{B}$ and $\mathrm{C}$ events. If it occurs, such pairs are discarded. When two negative event types are adjacent to each other, their order does not matter. For example, SEQ(A, !B, !C, D) is equivalent to $\operatorname{SEQ}(A, ! C, ! B, D)$. That is, all $(A, D)$ result pairs without any $B$ and $C$ events in between them would be returned. For negative event types at the end of a query, postponed sequence evaluation is applied. That is the execution is continued till the last negation as per our iterative strategy however results are not output. Instead at the arrival of every new event we note the time stamp of the event and also check whether it is a triggering event for the last negative part of the query. If it is not a triggering event, based on the time stamp of the arriving event, some results from the buffer may be output and removed from the buffer. If it is a triggering event, the negative part of the query is executed and if it produces some partial results, the result buffers of the outer query are completely cleared. However if the negative ending part of the query does not produce any results, some results are output and removed from the result buffers based on the time stamp of the arriving event.

In our nested query model, a sub-query as a whole could also be negated. For example, SEQ(A, ! AND(B, C), D). For each outer result of $\operatorname{SEQ}(\mathrm{A}, \mathrm{D})$, we search for $\mathrm{AND}(\mathrm{B}, \mathrm{C})$ results occurring between such $A$ and $D$ events. If none exist, then the outer SEQ(A, D) result is returned, otherwise it is filtered out.

We distinguish between the following positions in which the negation clause can occur.

- Bound by Upper Query. The existence of a negative event instance could be bounded by positive event instances in the direct upper queries. Examples of this category include $S E Q(A$, !B, C) and SEQ(A, SEQ(B, !C), D). In the second query, negative $\mathrm{C}$ events are bound by $\mathrm{B}$ and $\mathrm{D}$ events. $\mathrm{B}$ events that do not have any $C$ events occurring after them and before $D$ events are passed up to the upper query operator. All B events passed up will be joined with the outer SEQ(A, D) result to construct $\operatorname{SEQ}(A, \operatorname{SEQ}(B, ! C), D)$ results.

- Bound by Adjacent Query. The existence of a negative event instance could be bound by positive event instances of an adjacent sibling sub-query. Examples of this type include SEQ(A, SEQ(B, !C), SEQ(D, E), F) or SEQ(A, !B, SEQ(C, D), E). In this case, we apply a contextual delayed constraint technique. Namely, we conservatively pass up additional intermediate results as compared to the case described above. In SEQ(A, SEQ(B, !C), SEQ(D, E), F), outer SEQ(A, F) results $\left\langle a_{i}, f_{j}\right\rangle$ are constructed. The constraint window for both children sub-queries $\operatorname{SEQ}(B, ! C)$ and $\operatorname{SEQ}(D, E)$ is $\left[a_{i}\right.$.te, $f_{j}$.ts]. When processing the sub-query $\operatorname{SEQ}(\mathrm{B}, ! \mathrm{C})$ within this constraint window, any event of type $B$ should be passed up. We cannot filter out events of type B even though $\mathrm{C}$ events exist after it within its constraint window. The reason is that the right bound of the interval constraint of the query $\mathrm{SEQ}(\mathrm{B}, ! \mathrm{C})$ is decided by the results of the query SEQ(D, E). We should not have a $C$ event between a B or D event. However, it is not possible to know time stamps of $D$ events while still processing the query $\operatorname{SEQ}(B, ! C)$. Hence the decision is postponed until the results of both the inner queries are returned to the outer query and then the filtering of results takes place based on the presence of $\mathrm{C}$ events.

\subsection{Processing Nested Queries with Predicates}

The approach of handling sub-queries with correlated predicates is similar to the basic nested execution described above except that the join is not only based on timestamps but also on other predicates. Below, we list the different cases for predicate handling.

- Local predicates. Events are filtered based on predicate values before being stored in their stack. Query processing proceeds otherwise as explained above. For example, for the 
query in Figure 2, Operating events where the instrument type is not equal to "surgery" will be filtered.

- Correlated predicates between inner and outer queries. Nested sub-queries may be correlated with their parent queries by means of predicates. In order to evaluate these queries with predicates, it is necessary to pass down attribute values to the children queries. For example, the query in Figure 2 requires events in the inner sub-queries have the same tool id as the outer match. For each outer SEQ(Recycle r, Disinfection d, Operating o) match, the tool id information for the operating instance is thus passed down to the children sub-queries. Inner query results involving events having the same tool id with the outer match are returned to the upper query.

\subsection{Putting It All Together}

At compile time, queries with negation bounded by an adjacent sub-query (as discussed in Section 3.3) are marked with label "delayed constraint". More specifically, if a query $q_{i}$ is labeled as "delayed constraint", it not only needs to pass up potential $q_{i}$ results, but also negative events are passed up as we can't determine locally if they are in violation or not. The pseudo code of the nested execution algorithm is given in Figure 4. This function is called whenever a new event of the last positive event type in the outer query arrives. Figure 5 shows the algorithm for joining partial outer results with its children query results. As can be seen in Table 1, predicates on negative components are associated directly with the later and not with the operator a whole. They are thus only evaluated for those subqueries, for which the positive parent context match has already been established.

EXAMPLE 2. Consider the query $Q=S E Q($ Recycle $r$ ! SEQ( Washing $w$, Drying $d r$, Sharpening $s)$, Disinfection d, SEQ(Checking $c$, Relabeling $r l$ ), Operating op). When event instances of types Recycle, Washing, Drying, Sharpening, Disinfection, Checking, Relabeling and Operating arrive, they are pushed into their respective stacks. The outer query is first evaluated for a given window size followed by the inner sub-query. The outer query construction is triggered by the arrival of Operating events which are of the rightmost positive event type in the root query. For every partial result $<r_{i}, d_{j}$, op $_{k}>$ of the outer query SEQ(Recycle $r$, Disinfection $d$, Operating op), we compute the window constraints for its children queries. For details, see Figure 3. If we were to evaluate this query without predicates, all results for $S E Q$ (Washing w, Drying $d r$, Sharpening $s)$ and $S E Q($ Checking $c$, Relabeling $r l)$ would be constructed for events that occur within $\left[r_{i} . t e, d_{j} . t s\right]$ and $\left[d_{j} . t e\right.$, $\left.o p_{k} . t s\right]$, respectively. The outer operator joins with all results returned by its positive sub-query SEQ(Checking $c$, Relabeling rl). The outer result $\left\langle r_{i}, d_{j}, o p_{k}\right\rangle$ fails if results of the negative child query SEQ(Washing w, Drying dr, Sharpening s) exist. When evaluating $Q$ with correlated predicates [id], the id is passed down from the outer query to the children sub-queries. Results involving events with the same id are constructed in the sub-queries.

\section{PERFORMANCE EVALUATION}

The objective of our evaluation is to verify if our strategy gives the correct results so that they can be used as a benchmark to compare alternate future methods against. We verify using various types of queries. We also make note of the execution time to test the effectiveness and practicability of our method.

\subsection{Experimental Setup}

We have implemented our proposed nested query processing framework within the scalable complex event processing engine ECube [16] written in Java. We ran the experiments on Intel Pentium IV CPU $2.8 \mathrm{GHz}$ with $4 \mathrm{~GB}$ RAM. We evaluated our techniques using the real stock trades data from [17] with 10,000 event instances with a sliding window of size $10 \mathrm{~ms}$. The data contained stock ticker, timestamp and price information.

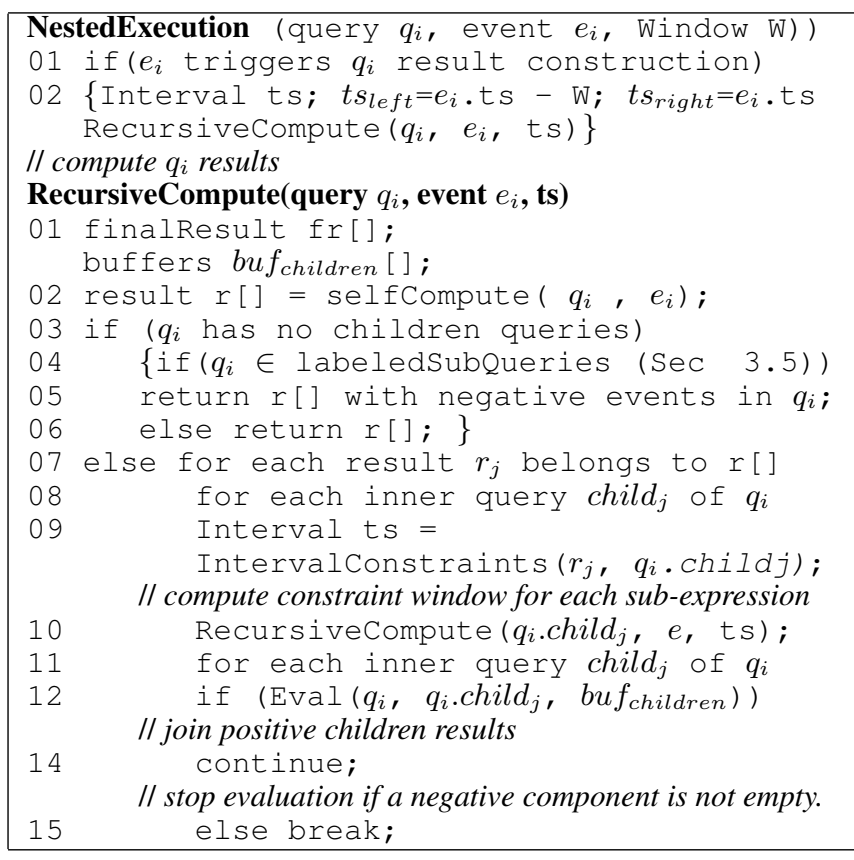

Figure 4: Nested Execution Strategy

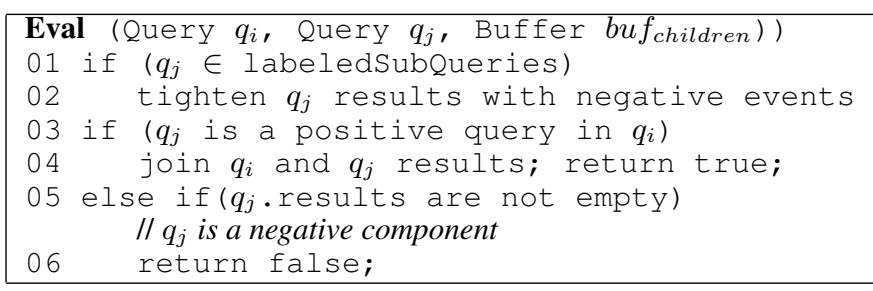

Figure 5: Result Evaluation

\subsection{Varying Children Subquery Number}

The first experiment processed queries with increased number of sub-queries from 1 to 3 (Figure 6(a)). $q_{3}$ generates minimum results using maximum processing time among the three queries. $q_{3}$ has more sub-queries to process which thus consumes more $\mathrm{CPU}$ processing time. Also, more outer SEQ(MSFT,ORCL,IPIX,INTC) results are filtered in $q_{3}$ as more constraints exist as compared to the other queries. As expected, the computation time increases with the number of sub-queries because the probability of finding patterns decreases with an increasing number of event types.

Increased Children Number:

$\mathrm{q} 1=\mathrm{SEQ}(\mathrm{MSFT}$, ! SEQ (RIMM, AMAT) , ORCL, IPIX, INTC);

q2=SEQ (MSFT, ! SEQ (RIMM, AMAT), ORCL, ! SEO (YHOO, DELL), IPIX, INTC) q3 $3=$ SEQ (MSFT, ! SEQ (RIMM, AMAT) , ORCL, ! SEQ (YHOO, DELL), IPIX, ! SEQ (CSCO, QQQ), INTC) ;

\subsection{Varying Subquery Lengths}

The second experiment processed the queries below with increased sub-query lengths (from 2 to 4 ) as depicted in Figure 6(b). We observed that $q_{6}$ generates the most number of results and uses the most CPU processing time among the three queries. This is because $q_{6}$ includes the sub-query with the longest length which consumes more computational time. As expected, less outer SEQ(MSFT, ORCL,INTC) results are filtered in $q_{6}$ as the existence of a longer pattern is relatively less likely as compared to the other queries with shorter patterns within the same input stream.

Increased Query Length:

$\mathrm{q} 4=\mathrm{SEQ}(\mathrm{MSET}, ! \mathrm{SEQ}$ (RIMM, AMAT), ORCL, INTC) ;

q5 5 SEQ $($ MSFT, ! SEQ (RIMM, AMAT, YHOO), ORCL, INTC);

$q 6=S E Q(M S F T, ! S E Q(R I M M$, AMAT, YHOO, DELL) , ORCL, INTC) ; 


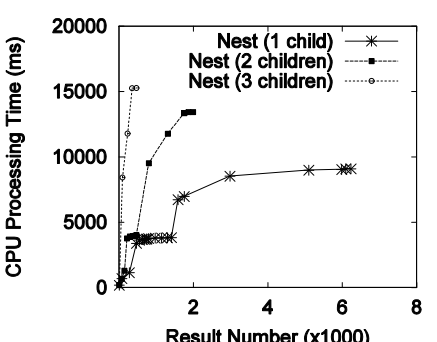

(a) Increased Children Number

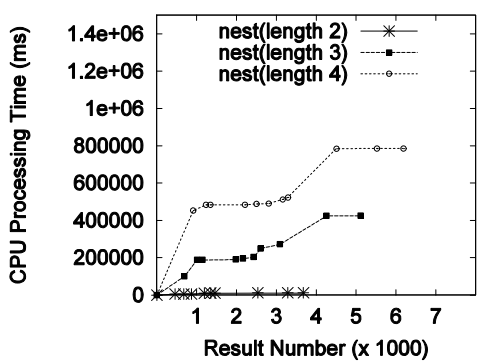

(b) Increased Query Length

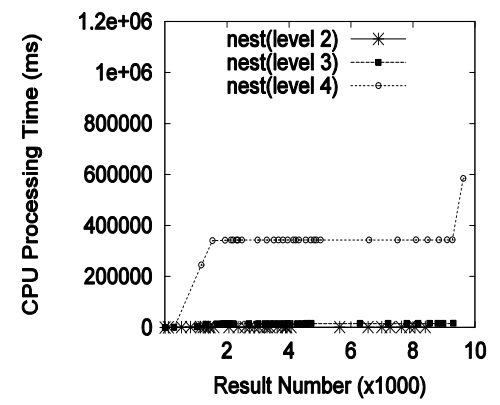

(c) Increased Nesting Levels

Figure 6: Evaluating Nested Patterns

\subsection{Varying Subquery Nesting Levels}

The third experiment processed the queries below with increased sub-query nesting levels as depicted in Figure 6(c). $q_{9}$ generates the most number of results and uses the most CPU processing time among the three queries. It is because $q_{9}$ includes the sub-query with the largest nesting levels which consumes more time to be computed. Less outer SEQ(MSFT, ORCL, INTC) results are filtered as it is relatively infrequent to have more events in levels occur in a sequence.

Increased Nesting Levels:

$q 7=\mathrm{SEQ}(\mathrm{MSFT}, ! \mathrm{SEQ}$ ( IPIX, QQQ) , ORCL, INTC)

$q 8=\mathrm{SEQ}(\mathrm{MSFT}, ! \mathrm{SEQ}($ IP IX, SEQ (RIMM, AMAT), $Q Q Q), \mathrm{ORCL}$, INTC) ; $q 9=$ SEQ $(M S F T, ! S E Q($ IPIX, SEQ (RIMM, SEQ (YHOO, DELL), AMAT), QQQ), ORCL, INTC);

\section{NESTED QUERY OPTIMIZATION}

Although the results of nested CEP queries obtained from the iterative execution strategy are correct, it produces results at a very slow rate which is attributed to the re-computation of the results for inner sub-queries every time an outer triggering event arrives which makes the processing expensive. To tackle this deficiency, we propose to cache and incrementally maintain the inner query results. Due to the sliding window, many intermediate results would continue to be valid from one sliding window to the next. Previously calculated results of the previous window should be cached and then be reused in the new window. In this paper we propose an optimization strategy of subexpression caching, focussing on positive nested subexpressions to explain the concept. We leave the refinement of this caching technique to support nested subqueries with negation or predicate correlation to our future work.

\section{Cache Design.}

We associate a cache with each query subexpression. Each cache is a triplet composed of the cache-interval, the output schema, and a container holding the set of cached results. The cache-interval is a semantic descriptor interval [leftbound,rightbound] that indicates the time validity of the current cache content. Put differently, the cache is guaranteed to contain the full set of result tuples valid in the [leftbound,rightbound] time range.

Cache Usage.

The same triggering event $e$ may generate multiple results for each subexpression with overlapping intervals. To avoid re-computation of results occurring in the same interval, we first extract the time interval that covers the time period for all the possible results triggered by the same event $e$ for a subexpression. Namely, the interval represents the minimum and maximum event time bounds for each subexpression. We will call this interval "query-interval". We will then check the "cache-interval". If there is an exact overlap between the two, we completely re-use the cached results. If however the "query-interval" is larger than the "cache-interval" we update the cache which we discuss in the next section.

Cache Maintenance.

Clearly, the cache content and associated semantic descriptor must be continuously maintained over time. We describe our strategies for cache maintenance below.

- Interval-driven Cache Expansion. We update the cache content when a new triggering event $e_{t}$ arrives. That is, given a new triggering event instance $e_{t}$, we calculate the new cache interval. For each subexpression, we compare the interval $[i, j]$ attached to the cache to the new interval $[\mathrm{m}$, $n$ ]. By the way our algorithm works, $i=m$, since the left bound is maintained at the event with minimum timestamp. We compute the sub-query $\operatorname{SEQ}\left(E_{i+1}, \ldots, E_{i+j}\right)$ for all triggering events $e_{i+j}$ between the interval $[j, n]$. New results are appended to the cache for each subexpression triggered by events.

- Interval-driven Cache Reduction. When a triggering event $e_{t}$ arrives, events with timestamp less than $e_{t}$ - window are purged from their stacks. Similarly, caching results involving events with timestamp less than $e_{t}$ - window are deleted from the cache as the window constraint will be violated if these results join with the new triggering event $e_{t}$ in the final result.

EXAMPLE 3. In Figure 7, when the triggering event $o_{26}$ arrives, it is inserted into the Operating stack and triggers execution. $[1,15]$ and $[8,26]$ are extracted time intervals for the subexpressions SEQ(Washing, Drying, Sharpening) and SEQ(Checking, Relabeling), respectively. SEQ(Washing, Drying, Sharpening) results are constructed based on all events that occurred during [1, 15]. Similarly, SEQ(Checking, Relabeling) events occurring during $[8,26]$ are constructed and cached. When the new triggering event $o_{30}$ arrives, we determine the interval for $S E Q$ (Washing, Drying, Sharpening) is still $[1,15]$. Thus the cache is still complete and thus we can reuse results in the cache. For subexpression $S E Q$ (Checking, Relabeling), we find the new interval [8, 30] overlaps with the previous interval $[8,26]$. Conceptually, we could reuse the caching results related to $[8,26]$ and we must compute the new additions to our cache. New SEQ(Checking, Relabeling) results are triggered by Relabeling events occurring between [26, 30] such as $\mathrm{rl}_{28}$. Assume the window size is 30 . When $o_{34}$ arrives, all caching results involving primitive events with time-stamp less than 4 expire. So $\left\langle w_{2}, d r_{6}, s_{7}\right\rangle,<w_{2}, d r_{3}, s_{7}>$ etc are deleted from the cache. The meta-data attached to the cache for SEQ(Washing, Drying, Sharpening) is updated from $[1,15]$ to $[4,15]$.

\subsection{Evaluating Optimized Nested Execution}

We process query $q_{10}$ comparing the optimized execution by the interval driven caching technique to the one without caching as in Figure 8. Caching helps in avoiding repeated computation for the subquery SEQ(QQQ,AMAT,DELL) as our results demonstrate. Clearly, we will have more or less gain with different reuse opportunities which may be caused by larger windows, more expensive sub-queries, etc. 


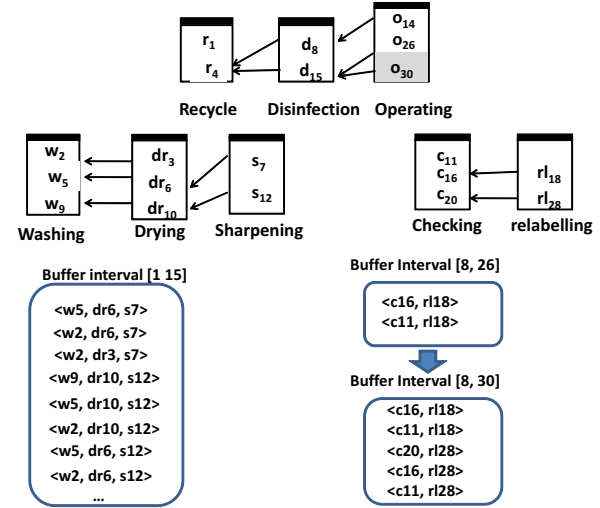

Figure 7: Interval Driven caching

Increased Nesting Levels:

q10 = SEQ (YHOO, SEQ (QQQ, AMAT, DELL), ORCL, IPIX);

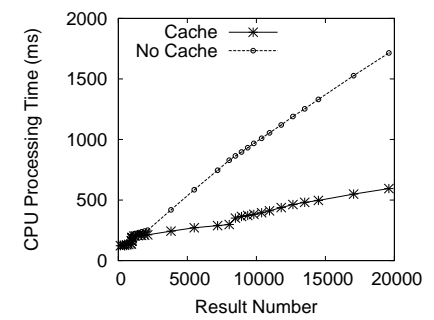

\section{RELATED WORK}

Figure 8: Interval-Driven Caching

The existing CEP systems $[1,2,3,6]$ do not focus on the execution of nested sequence queries as tackled in this paper. The query language of the CEDR [6] system supports nested sequence queries. However, the execution strategy for such nested queries is not given.

Nested iteration for SQL queries performs redundant work largely due to duplicate invocations of the correlated subquery with identical correlation values and interference with the locality of the outer query block. Query decorrelation [18, 19] techniques avoid such drawbacks, and aim to achieve set-based operations to further improve performance by exploiting customized physical altebra operators for query processing. Prior work in query decorrelation had focussed on SQL queries. The proposed iterative nested execution strategy for nested NEEL queries suffers from similar drawbacks - albeit in the context of CEP queries. To avoid duplicate subquery invocations, our nested query optimization technique selectively caches subquery results. Selective caching is appropriate in the context of streaming, where the inner query result cannot be completely computed apriori but rather its content is continuously changing over time due to the arrival of new and the purging of old events from the stream window. Further optimization such as techniques proposed in complex query decorrelation [18] will be considered in the future.

For SQL queries, [20] discusses whether a query result should be admitted to the cache and which results are to be purged in the static data context. In semantic caching [21], a semantic description of the data in a cache is maintained which allows for a compact specification. We study caching inner queries in the streaming context and apply interval driven caching by using validity intervals as semantic descriptors. Semantic descriptors have also be shown to be of importance for query caching in the XML context [22, 23]. However, sophisticated cache matching algorithms had to be designed to deal with query containment, namely, with extracting related yet not identical subexpressions possibly with alternate hierarchical XML structures yet the same content [22].

\section{CONCLUSION}

In this paper, we have introduced a comprehensive strategy for processing nested CEP queries expressed using the NEEL query language [7]. In particular, we introduce the window constraint tightening technique to correctly and efficiently process nested subqueries by restricting the set of events to be considered for the match. Our proposed iterative execution strategy considers both nested negation as well as predicates within nested subexpressions. Lastly, an optimization technique based on caching of positive subexpressions is introduced. Our preliminary experimental results on real data streams demonstrate the complexity of the iterative execution of nested queries, and show the effectiveness of applying our proposed optimization strategy to tackle this problem. Based on these promising results, we intend in the future to further refine this caching strategy as well as develop alternative techniques for the optimization of nested CEP queries.

\section{ACKNOWLEDGEMENTS}

This work is supported by HP Labs Innovation Research Program and National Science Foundation under grants NSF 1018443 and NSF IIS 0917017, Turkish foundation under TUBITAK career award 109E194. We also thank Database System Research Group members at WPI for their valuable comments.

\section{REFERENCES}

[1] E. Wu, Y. Diao, and S. Rizvi, "High-performance complex event processing over streams." in SIGMOD Conference, 2006, pp. 407-418.

[2] A. J. Demers, J. Gehrke, B. Panda, M. Riedewald, V. Sharma, and W. M. White, "Cayuga: A general purpose event monitoring system." in CIDR, 2007, pp. $412-422$.

[3] Y. Mei and S. Madden, "Zstream: a cost-based query processor for adaptively detecting composite events," in SIGMOD Conference, 2009, pp. 193-206.

[4] J. M. Boyce and D. Pittet, "Guideline for hand hygiene in healthcare settings," MMWR Recomm Rep., vol. 51, pp. 1-45, 2002.

[5] C. R. Baker and et al., "Wireless sensor networks for home health care."

[6] R. S. Barga, J. Goldstein, M. Ali, and M. Hong, "Consistent streaming through time: A vision for event stream processing." in CIDR, 2007, pp. 363-374.

[7] M. Liu, E. Rundensteiner, D. Dougherty, C. Gupta, S. Wang, and A. Mehta, "Neel: The nested complex event language for real-time event analytics," in BIRTE, 2010, pp. 358-369.

[8] S. Chakravarthy, V. Krishnaprasad, E. Anwar, and S.-K. Kim, "Composite events for active databases: Semantics, contexts and detection." in $V L D B, 1994$, pp. 606-617.

[9] A. J. Demers, J. Gehrke, B. Panda, M. Riedewald, V. Sharma, and W. M. White, "Cayuga: A general purpose event monitoring system." in CIDR, 2007, pp. $412-422$.

[10] J. Agrawal, Y. Diao, D. Gyllstrom, and N. Immerman, "Efficient pattern matching over event streams," in SIGMOD Conference, 2008, pp. 147-160.

[11] D. Gyllstrom, J. Agrawal, Y. Diao, and N. Immerman, "On supporting kleene closure over event streams," in ICDE, 2008, pp. 1391-1393.

[12] P. Seshadri, H. Pirahesh, and T. Y. C. Leung, "Complex query decorrelation," in ICDE, 1996, pp. 450-458.

[13] I. S. Mumick, S. J. Finkelstein, H. Pirahesh, and R. Ramakrishnan, "Magic is relevant," in SIGMOD Conference, 1990, pp. 247-258.

[14] E. Wong and K. Youssefi, "Decomposition - a strategy for query processing," ACM Trans. Database Syst., vol. 1, no. 3, pp. 223-241, 1976.

[15] J. M. Smith and P. Y.-T. Chang, "Optimizing the performance of a relational algebra database interface," Commun. ACM, vol. 18, no. 10, pp. 568-579, 1975

[16] M. Liu, E. A. Rundensteiner, K. Greenfield, C. Gupta, S. Wang, I. Ari, and A. Mehta, "E-cube: Multi-dimensional event sequence processing using concept and pattern hierarchies," in ICDE, 2010, pp. 1097-1100.

[17] "I. inetats. stock trade traces. http://www.inetats.com/."

[18] P. Seshadri, H. Pirahesh, and T. Y. C. Leung, "Complex query decorrelation," in ICDE '96: Proceedings of the Twelfth International Conference on Data Engineering. IEEE Computer Society, 1996, pp. 450-458.

[19] U. Dayal, "Of nests and trees: A unified approach to processing queries that contain nested subqueries, aggregates, and quantifiers," in $V L D B, 1987$, pp. 197-208

[20] J. Shim, P. Scheuermann, and R. Vingralek, "Dynamic caching of query results for decision support systems," in SSDBM, 1999, pp. 254-263.

[21] S. Dar, M. J. Franklin, B. T. Jónsson, D. Srivastava, and M. Tan, "Semantic data caching and replacement," in $V L D B, 1996$, pp. 330-341.

[22] L. Chen and E. A. Rundensteiner, "Xquery containment in presence of variable binding dependencies," in $W W W, 2005$, pp. 288-297.

[23] L. Chen, E. Rundensteiner, and S. Wang, "Xcache: A semantic caching system for xml queries," in ACM SIGMOD, 2002, pp. 618-618. 